\title{
Simulation von Innovationsdynamiken im Kontext der Energiewende
}

\author{
Beratungsorientierte Verbindung von Empirie und \\ sozialwissenschaftlichen simulationsmodellen
}

Joachim Globisch, Fraunhofer-Institut für System- und Innovationsforschung ISI, Breslauer Str. 48, 76139 Karlsruhe (joachim.globisch@isi.fraunhofer.de)

Bert Droste-Franke, IQIB - Institut für qualifizierende Innovationforschung und -beratung (bert.droste-franke@iqib.de)

Gabriele Fohr, IQIB - Institut für qualifizierende Innovationforschung und -beratung (gabriele.fohr@iqib.de)

Sandra Wassermann, Zentrum für interdisziplinäre Risiko- und Innovationsforschung (ZIRIUS), Universität Stuttgart (sandra.wassermann@zirius.uni-stuttgart.de)

Wie können Modelle zur Simulation von Akteursverhalten bei der Politikberatung zur Entwicklung von Maßnahmen zur Unterstützung der Genese und Diffusion nachhaltiger Technologien eingesetzt werden? Für Analysen zur Technologiegenese existiert bereits ein entsprechendes Simulationsmodell (SKIN). Mit Blick auf dieses Modell wird diskutiert, wie sich durch die Verbindung von Empirie und Simulation die Robustheit der Simulationsergebnisse steigern lässt. Für Akteursanalysen zur Technologiediffusion müssen entsprechende Simulationsmodelle erst noch entwickelt werden. Hierfür werden erste konzeptionelle überlegungen diskutiert.

\section{Simulation of innovation dynamics in the context of the energy transition \\ Advice-oriented combination of empirical and social science simulation models}

How can models for the simulation of actor behavior be used in policy advice on measures to support the development and diffusion of sustainable technologies? A corresponding simulation model already exists for analyses of technology development (SKIN). With reference to this model, it is discussed how the combination of empirical and simulation methods can increase the robustness of the simulation results. For actor analyses on technology diffusion, corresponding simulation models have yet to be developed. The article outlines some conceptual foundations for the development of such models.

Keywords: agent-based modeling, TIS analysis, empirical data, technology genesis, technology diffusion

This is an article distributed under the terms of the Creative Commons Attribution License CCBY 4.0 (https://creativecommons.org/licenses/by/4.0/)

https://doi.org/10.14512/tatup.28.3.34

Submitted: 08. 07.2019. Peer reviewed. Accepted: 24.10.2019

\section{Einleitung}

Im Zusammenhang mit der Energiewende bedarf es sowohl klassischer innovationspolitischer Maßnahmen zur Unterstützung der Technologiegenese als auch einer Förderung der Diffusion neuer Technologien (Weber und Rohracher 2012). Um die Effektivität solcher politischer Maßnahmen sicherzustellen, wird der Einsatz von Analyse- und Planungstools immer wichtiger. Im Bereich der Innovationspolitik bietet sich v. a. agentenbasierte Modellierung (ABM) als Simulationsinstrument an, um die Prozesse der Technologiegenese und -diffusion zu analysieren (s. hierzu auch TATuP 3/26 (2017)). Die Berücksichtigung von sozialwissenschaftlichen Ergebnissen in der Modellierung ist dabei von großer Bedeutung. So hängt z. B. die Diffusion neuer Energietechnologien stark von ihrer Akzeptanz durch Öffentlichkeit, potenzielle Nutzer*innen, Anwohner*innen, etc. ab (Wüstenhagen et al. 2007).

Dieser Artikel befasst sich daher mit methodischen Ansätzen, die die Evaluation von Politikmaßnahmen zur Förderung von Technologiegenese und -diffusion unter Berücksichtigung öffentlicher Unterstützung für diese Maßnahmen erlauben. Konzeptionelle Grundlage ist die Analyse Technologischer Innovationssysteme (TIS). Kerngedanke des TIS-Ansatzes ist, dass ein Innovationssystem aus Elementen (i.d. R. Organisationen, Institutionen, physische Infrastrukturen, Technologien) und Beziehungen zwischen den Elementen besteht (Hekkert und Negro 2011).

Nach Bergek et al. (2008) gliedert sich eine TIS-Analyse in sechs Schritte: (1) Definition des TIS, insbes. die „Flughöhe“ der Analyse (z. B. „Wärmepumpe“ vs. „Wärmetechnologien“); (2) Erfassung der drei übrigen zentralen Komponenten des TIS (Akteure, Netzwerke und Institutionen); (3) Analyse der Dynamik des TIS mit Blick auf sieben zentrale Funktionen (s. Tab. 1); 


\begin{tabular}{|c|c|}
\hline TIS-Funktion & Beschreibung \\
\hline 1. Unternehmerisches Experimentieren & Forschung, Anwendung, Testen und Kommerzialisierung von Wissen \\
\hline 2. Entstehung von Wissen & Lernen und schöpferische Anwendung von Wissen zur Entstehung neuen Wissens \\
\hline 3. Diffusion von Wissen & Austausch und Modifikation von Wissen \\
\hline 4. Suchrichtung & $\begin{array}{l}\text { Konvergenz der Zukunftsvorstellungen bzgl. Wachstum, technischer Entwicklung/Technikdesign } \\
\text { und übergeordneten Zielen des TIS }\end{array}$ \\
\hline 5. Ressourcenmobilisierung & Verfügbarkeit von Humankapital, politischer Unterstützung, finanziellem Kapital und Produktionsmitteln \\
\hline 6. Marktformierung & Herausbildung von Kundengruppen mit spezifischen Anforderungen und Kaufprozessen bzgl. des TIS \\
\hline 7. Legitimität & Unterstützung der neuen Technologie, Reduzierung von Unsicherheit \\
\hline
\end{tabular}

Tab.1: TIS-Funktionen.

Quelle: Eigene Darstellung nach Hekkert et al. 2007, Suurs 2009, Wieczorek et al. 2013, Bergek et al. 2008

(4) Einschätzung der Marktreife des TIS anhand der Dynamik der Funktionen; (5) Untersuchung von Anreiz- und Blockademechanismen (etwa durch etablierte Akteure und Technologien); (6) Ableitung von Handlungsempfehlungen aus den Schritten 1-5.

Obwohl die sieben Funktionen konzeptionell eine umfassende Charakterisierung eines TIS erlauben (s. Tab. 1), konzentrieren sich bisherige TIS-Analysen stark auf Untersuchungen zur Technologiegenese und damit auf die Funktionen 1 bis 3 . Um die Komplexität und Dynamik größerer gesellschaftlicher Transformationsprozesse bei TIS-Analysen besser zu verstehen, bedarf es einer stärkeren Berücksichtigung des Nachfragesystems sowie politischer und weiterer Akteure aus dem Umfeld des TIS (Weber und Rohracher 2012). Dazu kann konzeptionell an die Funktionen 4 bis 7 angeknüpft werden (vgl. Abb. 1).

So spiegelt die Funktion 6 „Marktformierung“ das Nachfragesystem, d.h. den Stand der Technologiediffusion, wider. Der Einfluss von Akteuren aus dem TIS-Umfeld kann über die Funktion 7 „Legitimität“ abgebildet werden, d.h. der Wahrnehmung und Unterstützung des TIS durch diese externen Akteure. Den Funktionen 4 ,Suchrichtung“ und 5 „,Ressourcenmobilisierung" kommt eine Brückenfunktion zwischen Technologiegenese und -diffusion zu: Je höher die Legitimität und je fortgeschrittener die Markformierung eines TIS ist, desto leichter können Ressourcen zu dessen (technologischer) Weiterentwicklung mobilisiert werden. Die Suchrichtung spiegelt die inhaltliche Konsolidierung eines TIS wider und steht in Wechselbeziehung zu den übrigen Funktionen. So fördert z. B. eine Konvergenz der Zukunftsvorstellungen zu einem TIS dessen Legitimität. Ähnliches gilt auch für die Zusammenhänge zwischen der Suchrichtung und den übrigen Funktionen.

Die beiden folgenden Kapitel knüpfen an diese Überlegungen zu den $\mathrm{Zu}-$ sammenhängen zwischen den TIS-Funktionen an. Im Kapitel Technologiegenese wird das Agentenmodell SKIN (Simula- ting Knowledge in Innovation Networks) vorgestellt. Hier wird erläutert, wie dieses Modell in Kombination mit empirischen Erhebungen zur Politikberatung mit Blick auf die Optimierung der drei Funktionen „Unternehmerisches Experimentieren“, „Entstehung von Wissen“ und „Diffusion von Wissen“ genutzt werden kann. Existierende Modelle zur Simulation der Technologiediffusion sind bisher techno-ökonomisch geprägt - Modelle zur Simulation von Akteursverhalten, müssen erst noch entwickelt werden. Daher befasst sich das Kapitel Technologiediffusion primär mit grundlegenden konzeptionellen Fragen zur Ausgestaltung eines solchen Akteursmodells und der Identifikation von Fragestellungen, zu deren Analyse sich das zu entwickelnde Akteursmodell in Kombination mit empirischen Erhebungen einsetzen lässt. Abschließend werden die Kernaussagen des Artikels zusammengefasst.

\section{Technologiegenese}

\section{Ansätze zur Simulation der Funktionen}

Um die zuvor dargestellten Funktionen bei der Technologiegenese simulieren zu können, wird das agentenbasierte Modell SKIN (Gilbert et al. 2010) herangezogen und um einige Funk-

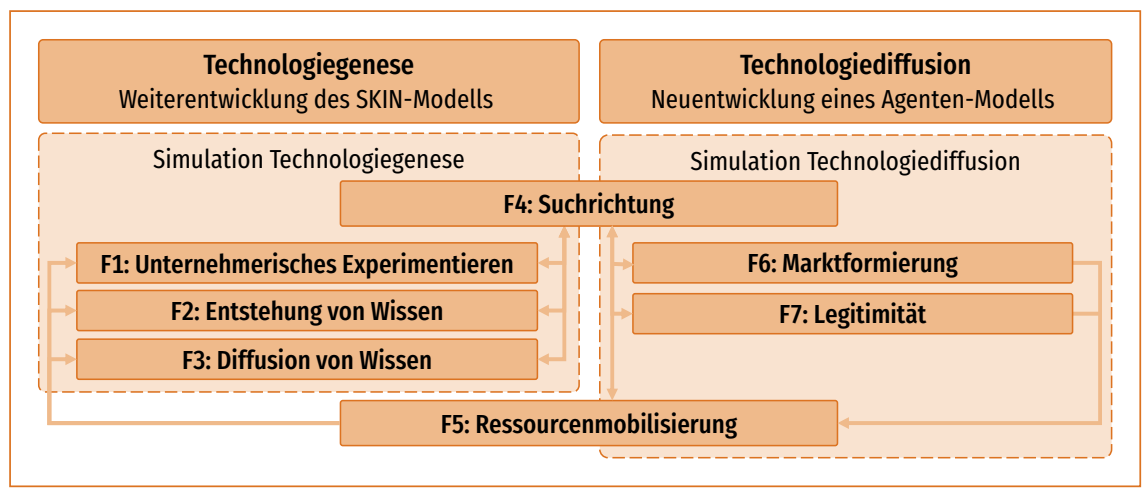

Abb.1: Zusammenhänge TIS-Funktionen, Technologiegenese und Technologiediffusion. 
tionalitäten erweitert, um es konkreter auf Technologieinnovationsprozesse anzupassen (Droste-Franke und Fohr 2017). Dieses wird im Folgenden ,erweitertes SKIN-Modell“ genannt.

Wissensgenese und -austausch sind zentrale Elemente von SKIN. In dem Modell interagieren Agenten mit heterogenen Eigenschaften in einer sich kontinuierlich verändernden Umgebung. Wissen kann entstehen aber auch vergessen werden und ein Produkt entsteht aus einer Kombination verschiedener Wissenskomponenten. Agenten im Modell sind vor allem Firmen und Forschungsinstitutionen, z. B. im Bereich der Entwicklung von Wärmepumpen. Firmen produzieren und verkaufen innova-

\section{Die Kombination von empirischen}

\section{Daten und ABM bietet mit Blick auf die Beratung zu möglichen Energiezukünften viele Vorteile.}

tive Güter, wie Wärmepumpen oder deren Vorprodukte. Dazu verwenden sie eigenes Wissen aber auch Wissen anderer Agenten. Dies umfasst zum einen Wissen aus Forschung und Entwicklung, aber auch praktisches Wissen für eine effiziente Produktion. Zum anderen werden geeignete Vorprodukte als Inputs anderer Agenten oder Ressourcen benötigt. Durch eigene Forschung und Forschung in Forschungsprojekten erhalten Firmen neues Wissen. Produktion und Forschung erhöht zudem die Expertise in vorhandenem Wissen. So können Wärmepumpen z. B. durch den Einsatz neuer Materialien oder eines neuen Designs effizienter gestaltet werden. Forschungsinstitutionen vermehren so ihr Wissen und tauschen es u. a. mit Firmen als Projektpartnern aus. Die Produkte werden auf dem Markt angeboten. Findet eine Wärmepumpe oder ein Vorprodukt keinen Absatz, senkt die Firma den Preis, forscht weiter oder wechselt das Produkt.

Um die Auswirkungen von Maßnahmen auf Struktur und Dynamik von Innovationsnetzwerken zu analysieren, werden diese im Rahmen von Simulationsexperimenten in SKIN implementiert. Maßnahmen können dabei direkt darauf abzielen, bestimmte Funktionalitäten des TIS zu verbessern. Eine Maßnahme könnte z. B. sein, die Zusammenarbeit und Kommunikation entlang der Wertschöpfungskette (z. B. zwischen Wärmepumpenherstellern und den Zulieferern) zu verbessern und damit den Wissensaustausch zwischen den jeweiligen Akteuren zu verstärken. Die TIS-Analyse kann dabei die Struktur vorgeben, in deren Rahmen Daten erhoben und Funktionalitäten bzgl. folgender Fragen bewertet werden: Ist eine geeignete Unternehmenslandschaft vorhanden um Wissensentstehung zu gewährleisten? Welche Arten von Wissen entstehen und wie wird Wissen ausgetauscht? Welche Hürden bestehen und wie können diese überwunden werden?

\section{Ansätze zur Verknüpfung von Empirie und Simulation}

Grundsätzlich kommen quantitative und qualitative Methoden der empirischen Innovationsforschung zum Einsatz, um mit SKIN realitätsnahe Abbildungen der Innovationsgenese zu ermöglichen. Einige empirisch erhobene Daten können direkt im Modell verwendet werden, z. B. empirische Ergebnisse zur Abstrahierung von Agenten und ihrer Umwelt, etwa indem die Anzahl von Patenten verschiedener Unternehmensklassen als Indikator für F \& E-Tätigkeiten mittels Patentanalyse zu Wärmepumpen erhoben werden. Solche Unternehmensklassen (z. B. nach Firmengrößen, Wirtschaftssektor, etc.) können dann als unterschiedliche Agententypen im Modell abgebildet werden. Informationen zur realen Zusammensetzung der Akteure eines TIS können aus Unternehmensstatistiken/-datenbanken gewonnen werden.

Zusätzlich zur Kalibrierung auf Mikroebene werden quantitative empirische Daten auf Meso- bzw. Makroebene herangezogen, mit denen sich die Ergebnisse der Modellierung vergleichen lassen. Durch Reproduktion beobachteter Strukturen in Innovationsnetzwerken, die sich etwa in beobachteten Forschungs- und Patentnetzwerken widerspiegeln, lässt sich die Validität des Modellierungsansatzes überprüfen.

Neben Ergebnissen aus quantitativen empirischen Analysen liefern auch qualitative Erhebungen wertvolle Informationen. Z. B. können in Interviews oder Workshops mit Wissenschaftler*innen und Praxisakteuren (1) die Funktionsmuster bei der Technologiegenese identifiziert, (2) die bisherige Funktionserfüllung bewertet, (3) Fragen an das Modell formuliert und (4) Modellergebnisse zur Ableitung von Handlungsempfehlungen diskutiert werden.

Tabelle 2 gibt einen Überblick über die Funktionen, die für die Technologiegenese besonders relevant sind sowie über Indikatoren und geeignete Datenquellen für empirische Erhebungen.

Im Modell sind des Weiteren allgemeinere Erkenntnisse über die Eigenschaften, Verhaltensweisen und strukturellen Rahmenbedingungen der handelnden Akteure abgebildet. Aus Studien ist beispielsweise bekannt, dass anwendbares Wissen je nach Kontext unterschiedlich intensiv ausgetauscht wird oder dass man Vorwissen in der Firma oder im Institut benötigt, etwa eine/n studierten Elektrotechniker*in, um bestimmtes Wissen aufnehmen und verwerten zu können. Auch die anderen Funktionen des TIS (s. u.) gehen u. a. so als Rahmenbedingungen ein.

\section{Simulationsexperimente mit SKIN zur Beratung}

Verschiedene Simulationsexperimente können mit dem Ziel der Beratung von (politischen) Entscheidungsträgern durchgeführt werden:

1. „Unternehmerisches Experimentieren“ ist im erweiterten SKIN durch Anwenden von Wissen zur Produktion von Gütern und durch die Gründung neuer Firmen auf verschiedene Weisen abgebildet. Der Erfolg wird durch die Erwirtschaftung von Erlösen abgebildet. Durch Simulationsexperimente können die Auswirkungen auf das Innovationssystem von Änderungen der Rahmenbedingungen für diese Aktivitäten 


\begin{tabular}{|c|c|c|}
\hline TIS-Funktion & Indikatoren/Event-/Ereignistypen & Daten/Datentypen, Quellen (Auswahl) \\
\hline $\begin{array}{l}\text { Unternehmerisches } \\
\text { Experimentieren }\end{array}$ & $\begin{array}{l}\text { Anzahl Akteure, Anzahl Unternehmen, Anzahl Entrepreneure, } \\
\text { neue Sparten etablierter Anbieter, Spezialisierung entlang } \\
\text { der Wertschöpfungskette }\end{array}$ & Unternehmens-, Branchenstatistik(en) \\
\hline Entstehung von Wissen & $\begin{array}{l}\text { Anzahl Patente, Publikationen, F \& E-Projekte, Forschungs- } \\
\text { intensität(en); Lernkurven }\end{array}$ & $\begin{array}{l}\text { Patent- und F \& E-Statistiken (EPO PatStat, Förderkatalog, } \\
\text { Wissenschaftsstatistik) }\end{array}$ \\
\hline Diffusion von Wissen & $\begin{array}{l}\text { Netzwerkgröße und -dichte, gemeinsame F \& E-Projekte, } \\
\text { gemeinsam gehaltene Patente, Zitationen, Workshops, Messen, } \\
\text { Konferenzen }\end{array}$ & $\begin{array}{l}\text { Patent- und F\& E-Statistiken, Mitglieder Branchenverbände, } \\
\text { Teilnehmer Konferenzen und Workshops }\end{array}$ \\
\hline Suchrichtung & $\begin{array}{l}\text { Anzahl und Gewicht wissenschaftlicher und journalistischer } \\
\text { Publikationen, Zielsetzungen, Regierungs- oder Förderprogramme }\end{array}$ & $\begin{array}{l}\text { H-Index von Publikationen, Patenten, Themen von Forschungs- } \\
\text { programmen }\end{array}$ \\
\hline
\end{tabular}

Tab.2: TIS-Funktionen und empirische Belege.

Quelle: Eigene Darstellung basierend auf Wieczorek et al. 2013, Hekkert et al. 2007

getestet werden, z. B. Zahl neuer Produkte bzw. Zahl von Start-ups verschiedener Typen.

2. „Die Entstehung von Wissen“ erfolgt im erweiterten SKIN durch Forschung und neue Akteure. Die Abbildung erfolgt durch einen evolutionären Prozess. Dem Wissen eines Akteurs ist immer auch eine Expertise zugewiesen. Diese erhöht sich für Wissen, das zur Produktion eingesetzt wird mit der Zahl der Produktionszyklen und bei Wissen, das in Projekte einfließt, mit seiner Verwendung in den Projekten. Entsprechend können mit dem Modell Auswirkungen von Änderungen in Rahmenbedingungen für unternehmerische und wissenschaftliche Forschung untersucht werden, die sich z. B. durch Schwerpunkte von Forschungsprogrammen ergeben.

3. Wissensaustausch zwischen den Akteuren erfolgt im erweiterten SKIN im Rahmen von bilateralen Zusammenarbeiten bei der Produktion, in Produktionsnetzwerken und bei Zusammenarbeiten in Forschungsprojekten. Dabei werden praktisches und wissenschaftliches Wissen unterschieden, Unterschiede in der Aufnahmemöglichkeit von Wissen abhängig vom Vorwissen berücksichtigt und Zuwachs von Expertise in verschiedenen Wissensbereichen modelliert, abhängig von der Menge des erhaltenen Wissens. Mit dem Modell können unter anderem Auswirkungen von Maßnahmen (wie spezifische Anreize zur Zusammenarbeit, Etablierung von Firmennetzwerken, Etablierung neuer Akteure mit entsprechenden Aufgaben und Veranstaltungsformaten, z. B. Energieagenturen) simuliert werden, die die Intensität des Wissensaustausches zwischen verschiedenen Akteuren (z. B. entlang/quer zur Wertschöpfungskette) oder die Erhöhung der Aufnahmefähigkeiten fördern.

4. Anleitung zur Suche ist im Modell nicht detailliert abgebildet, sondern durch Rahmenbedingungen vorgegeben. Eine Veränderung der Rahmenbedingungen, wie z. B. eine spezifischere Ausrichtung von Projektausschreibungen auf konkrete Wissensbereiche, führt über die Modelldynamik zu anderem Wissen, anderen Strukturen und Dynamiken. Auch Auswirkungen marktlicher Anreize über Nachfrage- oder Entwicklungs- und Produktionsförderung können im Modell simuliert werden.

\section{Technologiediffusion}

Für Analysen zur Technologiediffusion sind derzeit noch keine dem SKIN-Modell vergleichbaren Simulationstools vorhanden. Daher werden nachfolgend zunächst grundlegende konzeptionelle Aspekte der Frage adressiert, welche Aktivitäten von welchen Akteuren in Simulationstools zur Technologiediffusion abgebildet werden sollten, um einen Mehrwert bei der Politikberatung zu erbringen.

\section{Konzeptionelle Grundlage von Diffusionsanalysen}

Die Marktformierung in einem TIS kann als Ausdruck der Technologiediffusion betrachtet werden und Legitimität bildet für letztere eine zentrale Voraussetzung. Die Dynamik beider Funktionen hängt stark von Entscheidungen individueller Akteure ab: Konsument*innen treffen Kaufentscheidungen, in Unternehmen bedürfen neue Technologien der Unterstützung von sog. Innovation Champions (Globisch et al. 2018) und bei Politikmaßnahmen stellt sich die Frage nach der Akzeptanz von Bürger*innen sowie Betroffenen. Daher scheint eine Verbindung von TIS-Analyse und Akzeptanzforschung, die konzeptionell stärker auf die Analyse von individuellen Entscheidungen abzielt, sinnvoll. Hinsichtlich individueller Akteure können in Anlehnung an Wüstenhagen et al. (2007) drei Akzeptanzformen unterschieden werden (Bezug zu Abb. 2 jeweils in Klammern):

1. Sozio-politische Akzeptanz (I.): Die allg. Befürwortung oder Ablehnung von Technologien (z. B. Wärmepumpen) und Politikmaßnahmen (z. B. Verbote konventioneller Heizungen) durch die Wähler*innen.

2. Marktakzeptanz (II.): Die Adoption einer Technologie durch Entscheider*innen in Haushalten und Unternehmen als Nachfrager oder Investoren (z. B. Entscheidung für/gegen den Einbau einer Wärmepumpe).

3. Lokale Akzeptanz (III.): Die Reaktion von vor Ort konkret Betroffenen auf die Umsetzung von Adoptionsentscheidungen (z. B. Mieter*innen) und Politikmaßnahmen (z. B. Bauleitungen). 
F1-F3: Technologiegenese in Forschung und Angebotssystem

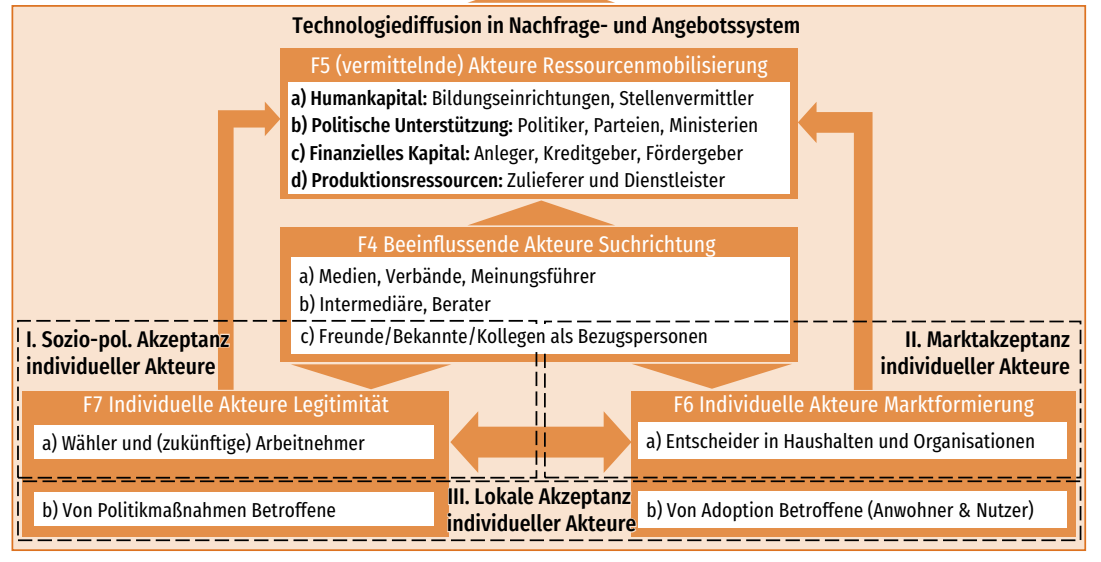

Abb.2: Technologiediffusion in Nachfrage- und Angebotssystem; Abweichungen zu Abb.1 ergeben sich aus dem primären Fokus auf individuelle Akteure.

Quelle: Eigene Darstellung in Anlehnung an Dütschke et al. 2019

von Legitimität $(\mathrm{F} 4 \mathrm{a} / \mathrm{b} \rightarrow \mathrm{F} 7)$ und Adoptionsentscheidungen $(\mathrm{F} 4 \mathrm{a} / \mathrm{b} \rightarrow \mathrm{F} 6)$ ?

Mit Blick auf die Ressourcenmobilisierung zur Unterstützung der Technologiegenese (F4 $\rightarrow$ F1-F3) lassen sich weitere Fragestellungen zum Einfluss individueller Akteure auf vermittelnde Akteure ableiten; z. B. welchen Einfluss hat die Legitimität eines TIS auf Studien- und Ausbildungsentscheidungen und die Ausgestaltung von Lehrplänen (z. B. Attraktivität von Bauingenieurs-Studiengängen mit Fokus auf Wärmepumpen/alternative Heiztechnologien; F7 a $\rightarrow$ F5 a)? Wie hängt die Legitimität eines TIS aus Sicht von Bürgern und Betroffenen mit der Positionierung von politischen Akteuren zusammen (F7a/b $\rightarrow$ F5b)? Inwieweit werden Entscheider bei potenziellen Kapitalgebern, Zulieferern und Dienstleistern in ihrer Wahrnehmung der TIS-Entwicklung auch durch persönliche Erfah-

\section{Fragestellungen an Diffusionsanalysen}

Ein Fokus auf individuelle Akteure führt zu einer entsprechenden Perspektive auf die Entstehung von Legitimität und Marktformierung, aus der sich unter anderem folgende Fragestellungen ergeben:

Entstehung Legitimität (F7): Unter welchen Voraussetzungen kann sich ein entstehendes TIS als förderungswürdige Alternative zu einem bestehenden TIS legitimieren, obwohl evtl. Nachteile hinsichtlich Kosten, Komfort, o. ä. bestehen (z. B. Wahrnehmung von Wärmepumpen als sinnvolles Element der Wärmewende; F7 a)? Welchen Einfluss hat die Legitimität auf die lokale Akzeptanz von Fördermaßnahmen für ein TIS (z. B. Akzeptanz von Vorschriften zur Heiztechnologie in Bebauungsplänen; F7 b)?

Dynamik Marktformierung (F6): Welchen Beitrag können Modelle zu Adopter-Gruppen (Rogers 2003) und psychologische Theorien zu individuellen (Adoptions-)Entscheidungen zu einem beratungsorientierten Verständnis der Markformierung leisten (z. B. Einflussfaktoren auf Beschaffung von Wärmepumpen; F6a)? Inwieweit sind Dritte von Adoptionsentscheidungen betroffen und spielen dadurch als Akteur*innen bei der Marktformierung eine Rolle (z. B. Akzeptanz von Umlage der Investitionskosten für eine neue Wärmepumpe durch die Mieter*innen; F6 b)?

Einfluss Suchrichtung (F4): Welchen Einfluss hat das persönliche Umfeld auf die Meinung von Bürger*innen (z. B. Einfluss von Meinungsführer*innen auf wahrgenommene Legitimität von Wärmepumpen ggü. anderen Heiztechnologien; F4 c $\rightarrow$ F7a) und potenziellen Adoptern (Einfluss von Bekannten auf wahrgenommene Attraktivität von Wärmepumpen ggü. anderen Heiztechnologien; F4c $\rightarrow$ F6a)? Welchen Einfluss haben gesellschaftliche Akteure und Intermediäre auf die Entstehung rungen mit einer Technologie (F6 $\rightarrow$ F5 c/d) oder persönliche Werte und Einstellungen (F7 $\rightarrow$ F5 c/d) beeinflusst?

\section{Mögliche empirische Grundlagen zur Simulation der Technologiediffusion}

Für die Untersuchung dieser Fragen können qualitative und quantitative Primärdaten erhoben werden. Weiterhin können auch Prozessdaten nützliche Datenquellen sein. So können bspw. Social-Media-Beiträge Einblicke in die Entstehung und Diffusion von Meinungen und Einstellungen erlauben. Darüber hinaus kann auch die Analyse von Medien-Berichten, Pressemitteilungen oder Parlamentsprotokollen u. ä. für die Identifikation und Positionsbestimmung der beeinflussenden gesellschaftlichen Akteure nützlich sein (Tab. 3). Solche Analysen können wiederum Ausgangspunkt für die Untersuchung des Einflusses dieser Akteure auf individuelle Einstellungen und Entscheidungen sein, die die Grundlage von Legitimität und Marktformierung bilden.

Zur Analyse der empirischen Daten können verschiedene Verfahren verwendet werden, die die Untersuchung unterschiedlicher Teilaspekte der genannten Forschungsfragen erlauben. Beispielhaft seien hier vier Verfahren genannt: (1) Strukturgleichungsmodelle zur Analyse des Zusammenspiels (psychologischer) Eigenschaften von Individuen und deren Einfluss auf die Akzeptanz von Maßnahmen und Technologien; (2) Choice- und Vignetten-Experimente zur Analyse des Einflusses der Eigenschaften von Technologien oder Maßnahmen auf deren Akzeptanz; (3) Clusteranalytische Verfahren zur Identifikation von Subgruppen (z. B. bei den Ergebnissen der zuvor genannten Verfahren); (4) (Egozentrische) Netzwerkanalysen zur Untersuchung der Struktur von interpersonellen Netzwerke und ihres Einflusses auf Einstellungen und Entscheidungen. 


\begin{tabular}{|c|c|c|}
\hline TIS-Funktion & Indikatoren/Event/Ereignistypen & Daten/Datentypen/Quellen (Auswahl) \\
\hline Suchri & $\begin{array}{l}\text { Positionierung beeinflussender Akteure zu TIS Mobilisierungsfähigkeit beeinflussender } \\
\text { Akteure bzgl. TIS }\end{array}$ & \multirow{4}{*}{$\begin{array}{l}\text { - Bei Analysen zu beeinflussenden und vermit- } \\
\text { telnden (kollektiven) Akteuren: Text- und Social- } \\
\text { Discourse-Analyse von/mit Medienberichten, } \\
\text { Parlamentsprotokollen, Pressemitteilungen u. Ä. } \\
\text { - Qualitative Interviews, Gruppendiskussionen } \\
\text { und (Experten-)Workshops } \\
\text { - Quantitative Quer- und Längsschnitt- } \\
\text { befragungen (Auswertung z. B. mit u. g. Analyse- } \\
\text { methoden 1-4) } \\
\text { - Analyse von Inhalt und Zusammenhängen } \\
\text { von Social-Media-Beiträgen }\end{array}$} \\
\hline $\begin{array}{l}\text { Ressourcen- } \\
\text { mobilisierung }\end{array}$ & $\begin{array}{l}\text { Politische Unterstützung: Positionierung politischer Akteure im gesellschaftlichen } \\
\text { Diskurs zum TIS } \\
\text { Humankapital: Attraktivität des TIS für Arbeitnehmer*innen; TIS-bezogene } \\
\text { Bildungsangebote } \\
\text { Finanzielles Kapital und Produktionsmittel: Attraktivität des TIS als Geschäftsfeld }\end{array}$ & \\
\hline Marktformierung & $\begin{array}{l}\text { Marktakzeptanz: (Intention zu) Technologieadoption } \\
\text { Lokale Akzeptanz: Akzeptanz der Technologieadoption durch Betroffene }\end{array}$ & \\
\hline Legitimität & $\begin{array}{l}\text { Sozio-politische Akzeptanz: Positive Einstellung der Bürger*innen zu Technologie/TIS } \\
\text { Lokale Akzeptanz: Akzeptanz von Politikmaßnahmen durch Betroffene }\end{array}$ & \\
\hline
\end{tabular}

Tab.3: TIS-Funktionen und empirische Belege.

Quelle: Eigene Darstellung

\section{Verbindung von Empirie und Simulation}

Der Einsatz von ABM in Verbindung mit empirischen Analysen zur Untersuchung der genannten Forschungsfragen bietet sich v. a. aus zwei Gründen an:

1. Mit Blick auf die Beratung von (politischen) Entscheider*innen bietet der Einsatz von ABM vor allem den Vorteil, dass Szenarien zu möglichen Zukünften unter verschiedenen Annahmen simuliert und dadurch veranschaulicht werden können.

2. Im Rahmen einzelner empirischer Untersuchungen können stets nur Teilaspekte der Technologiediffusion adressiert werden. Durch den Einsatz von ABM können die Ergebnisse verschiedener empirischer Analysen zusammengeführt und so die Komplexität der Technologiediffusion besser berücksichtigt werden.

Damit eine Verbindung unterschiedlicher empirischer Untersuchungen mittels ABM gelingen kann, sind aber insbesondere zwei Herausforderungen zu bewältigen: (1) Damit Ergebnisse empirischer Analysen passfähig zur Struktur und Funk- nicht möglich. Daher bedarf es der (empirischen) Identifikation oder (zweckmäßigen) Definition von Subgruppen, über die die empirischen Ergebnisse unterschiedlicher Erhebungen den Agenten einer ABM zugeordnet werden können.

\section{Zusammenfassung und Schlussfolgerungen}

Aus den vorherigen Kapiteln wird deutlich, dass die Kombination von empirischen Daten und ABM mit Blick auf die Beratung $\mathrm{zu}$ möglichen Energiezukünften viele Vorteile bietet. ABM erlaubt die Simulation von Vorgängen in und zwischen Akteuren auf der Mikroebene und die Aggregation der Folgen dieser Vorgänge auf die Makroebenen. Durch die Simulation auf der Mikroebene kann die Beratung zu Politikmaßnahmen die (Heterogenität der) Entscheidungsprozesse von Adressaten und Stakeholdern berücksichtigen. Zugleich sind mittels Wenn-dann-Analysen Aussagen zu möglichen Folgewirkungen von unterschiedlich ausgeformten Politikmaßnahmen auf der Makroebene möglich. Darüber hinaus bietet die Simulation der

\section{ABM erlaubt die Simulation von Vorgängen in und zwischen Akteuren auf der Mikroebene und die Aggregation der Folgen dieser Vorgänge auf die Makroebenen.}

tionsweise einer ABM sind, sollten sich beide inhaltlich an den gleichen theoretischen Modellen orientieren. Dafür bedarf es möglichst universell anwendbarer theoretischer Modelle bzw. Meta-Modelle, die unterschiedliche theoretische Modelle miteinander verbinden. (2) Da in unterschiedlichen empirischen Untersuchungen auch unterschiedliche Personen befragt werden, ist eine Zusammenführung der Daten auf individueller Ebene
Interaktion zwischen verschiedenen Akteuren die Möglichkeit, auch nicht-intendierte Folgen einer Maßnahme zu erkennen, die sich aus zuvor nicht bedachten Reaktionen und Rückkopplungseffekten ergeben können.

Mit Blick auf die Kombination von Empirie und Simulation wurde gezeigt, wie verschiedene qualitative und quantitative Datenquellen (Tab. 2) zur Validierung und Kalibrierung des 
SKIN-Modells genutzt werden können, um robuste Systemanalysen zur Politikberatung zu ermöglichen. Zudem erlaubt die empirische Fundierung eine Simulation möglicher Entwicklungsverläufe, die einige Jahre in die Zukunft reichen. Durch die Berücksichtigung der Technologiediffusion und des gesellschaftlichen Umfelds eines TIS kann die Aussagekraft von ( $\mathrm{Si}$ mulations-)Ergebnissen zu möglichen Zukünften gesteigert werden. Auch hier bietet sich eine Kombination von Empirie und ABM als methodischer Ansatz zur Politikberatung an. Im $\mathrm{Zu}$ sammenhang mit der Analyse der Technologiediffusion sind die Entscheidungen individueller Akteure von besonderer Bedeutung, da sie der Entstehung von Legitimität, der Dynamik der Markformierung und der Mobilisierung wichtiger Ressourcen zugrunde liegen.

Die Verknüpfung beider Modelle und damit die Abbildung aller Funktionen des TIS stellt eine vielversprechende Möglichkeit dar, ein Innovationssystem in seiner Komplexität besser zu modellieren und zu untersuchen. Der Bedarf solcher kombinierten Analysen ergibt sich daraus, dass das Gelingen der Energiewende sowohl von der Dynamik technologischer Entwicklungen als auch von deren Akzeptanz abhängt.

\section{Literatur}

Bergek, Anna; Jacobsson, Staffan; Carlsson, Bo; Lindmark, Sven; Rickne, Annika

(2008): Analyzing the functional dynamics of technological innovation

systems. A scheme of analysis. In: Research Policy 37 (3), S. 407-429.

Dütschke, Elisabeth; Bögel, Paula; Choi, Su-Min; Globisch, Joachim; Burghard,

Uta (2019): Soziale Akzeptanz als erweitertes Verständnis des Akzeptanz-

begriffs. Eine Bestimmung der Akteure für den Prozess der Energiewende.

In: Akzeptanz und politische Partizipation in der Energietransformation.

Wiesbaden: Springer VS, S. 211-230.

Droste-Franke, Bert; Fohr, Gabriele (2017): Simulating innovation of key techno-

logies in German energy transition. Social Simulation Conference (SSC).

Dublin, Irland, 25-29.09.2017.

Gilbert, Nigel; Ahrweiler, Petra; Pyka, Andreas (2010): The SKIN (Simulating

Knowledge Dynamics in Innovation Networks) model. University of Surrey, University College Dublin, University of Hohenheim, https://github.com/ InnovationNetworks/skin, zuletzt geprüft am 23.10.2019.

Globisch, Joachim; Dütschke, Elisabeth; Wietschel, Martin (2018): Adoption of electric vehicles in commercial fleets. Why do car pool managers campaign for BEV procurement? In: Transportation Research Part D: Transport and Environment 64, S. $122-133$.

Hekkert, Marko; Negro, Simona (2011): Understanding technological change. Explanation of different perspectives on innovation and technological change. Utrecht University. Online verfügbar unter https://pdfs. semanticscholar.org/34c9/22f3580c98c2ccf50818a70525652fdd3b5e.pdf, zuletzt geprüft am 21.10.2019.

Hekkert, Marko; Suurs, Roald; Negro, Simona; Kuhlmann, Stefan; Smits, Ruud (2007): Functions of innovation systems. A new approach for analysing technological change. In: Technological Forecasting \& Social Change 74 (4), S. 413-432.

Suurs, Roald (2009): Motors of sustainable innovation. Towards a theory on the dynamics of technological innovation systems. Dissertation Utrecht University.
Weber, Matthias; Rohracher, Harald (2012): Legitimizing research, technology and innovation policies for transformative change. In: Research Policy 41 (6), S. 1037-1047.

Wieczorek, Anna; Negro, Simona; Harmsen, Robert; Heimeriks, Gaston; Luo, Lin; Hekkert, Marko (2013): A review of the European offshore wind innovation system. In: Renewable and Sustainable Energy Reviews 26, S. 294-306.

Wüstenhagen, Rolf; Wolsink, Maarten; Bürer, Mary (2007): Social acceptance of renewable energy innovation. An introduction to the concept. In: Energy Policy 35, S. 2683-2691.

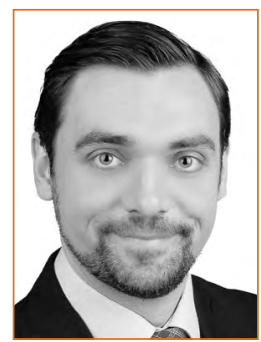

\section{DR. JOACHIM GLOBISCH}

forscht seit 2012 am Fraunhofer ISI zur Akzeptanz von Energietechnologien. Sein methodischer Schwerpunkt liegt auf quantitativen empirischen Untersuchungen und der Verbindung von Empirie und Modellierung.

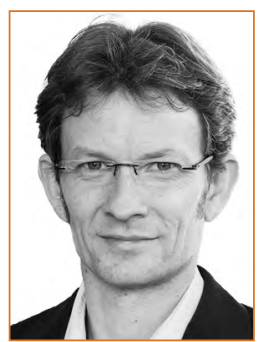

\section{DR. BERT DROSTE-FRANKE}

ist Head of Department Energy beim IQIB. Seine Schwerpunkte sind System-, Innovations- und Technikfolgenanalysen zur Entscheidungsunterstützung auf Basis theoretischer Arbeiten, inter-/ transdisziplinärer Expertengruppen, quantitativen Analysen und Modellierungen.

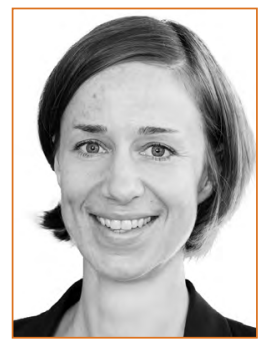

\section{GABRIELE FOHR}

ist wissenschaftliche Mitarbeiterin im Department Energy des IQIB. Ihr Hauptarbeitsfeld liegt in der Methodenentwicklung, vor allem für die Energiesystemanalyse, sowie in der Resilienzforschung.

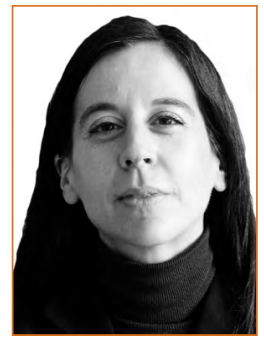

\section{SANDRA WASSERMANN}

ist bei ZIRIUS, Universität Stuttgart beschäftigt. Seit 2016 ist sie dort Sprecherin des Forschungsbereichs Energie. Ihr Forschungsinteresse gilt der Analyse von technologischen und sozialen Innovationen im Zuge der Energiewende. 